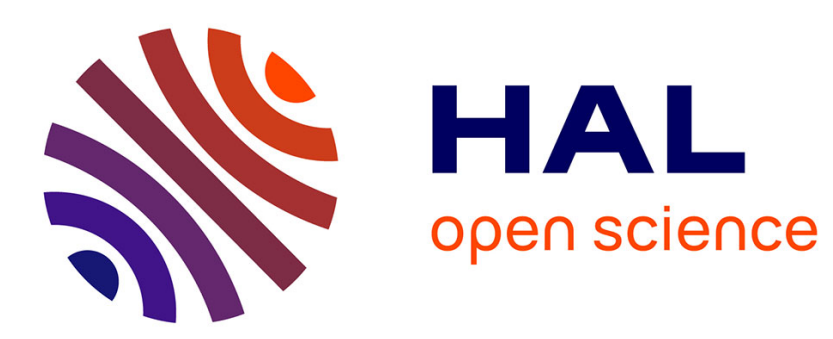

\title{
Finite Elements Simulation of Mechanical Behaviour of Shape Memory Alloys Coupled with a Non-Stationary Thermal Field
}

\author{
G. Rio, D. Favier, H. Desplats
}

\section{- To cite this version:}

G. Rio, D. Favier, H. Desplats. Finite Elements Simulation of Mechanical Behaviour of Shape Memory Alloys Coupled with a Non-Stationary Thermal Field. Journal de Physique IV Proceedings, 1995, 05 (C8), pp.C8-215-C8-220. 10.1051/jp4:1995829 . jpa-00254078

\section{HAL Id: jpa-00254078 https://hal.science/jpa-00254078}

Submitted on 1 Jan 1995

HAL is a multi-disciplinary open access archive for the deposit and dissemination of scientific research documents, whether they are published or not. The documents may come from teaching and research institutions in France or abroad, or from public or private research centers.
L'archive ouverte pluridisciplinaire HAL, est destinée au dépôt et à la diffusion de documents scientifiques de niveau recherche, publiés ou non, émanant des établissements d'enseignement et de recherche français ou étrangers, des laboratoires publics ou privés. 


\title{
Finite Elements Simulation of Mechanical Behaviour of Shape Memory Alloys Coupled with a Non-Stationary Thermal Field
}

\author{
G. Rio, D. Favier* and H. Desplats \\ LG2M, Université de Bretagne Sud, CGI, Guidel-Plages, 56520 Guidel, France \\ * 3S, URA 1511 du CNRS, Université J. Fourier, BP. 53X, 38041 Grenoble cedex, France
}

\begin{abstract}
The main deformation mechanisms of shape memory alloys (SMA) are related to martensitic transformation and/or martensitic reorientation. These phenomena lead to heat production resulting from the transformation and/or from internal dissipation processes. As the SMA behaviour is strongly temperature dependent, its modelling has to take into account thermomechanical coupling. A 3-dimensional finite element code has been designed for that purpose. The quasi-static mechanical behaviour is described through the temperature dependent elastohysteresis model. Large displacements and large deformations are considered. The numerical problem is strongly non-linear and an implicit resolution scheme has been adopted. Two coupled thermomechanical calculations are presented. The first one deals with the one-way memory effect of a cantilever beam. The second one simulates the non-stationary thermal and mechanical fields of this beam during a near-adiabatic pseudoelastic bending.
\end{abstract}

\section{INTRODUCTION}

Deformation processes of any solid body represent thermomechanical processes. This is of particular importance for shape memory alloys (SMA) whose deformation mechanisms are mainly related to martensitic transformation and variant reorientation. The thermal effects are obviously to be taken into account for SMA when an alloy deformed in its martensitic phase by martensite reorientation recovers its initial shape when heated into the stable austenitic state. In that case the thermomechanical coupling is increased by significant generation of heat during the martensitic and reverse transformation. For a temperature greater than the austenite finish temperature (Af) the interplay of stress and temperature leads also to the pseudoelastic behaviour for which the stressing of initial austenite results in a large straining of the material due to the exothermic forward transformation. The occurrence of the endothermic reverse transformation during unloading leads to the recovering of the initial shape. In that case, the mechanical behaviour can be significantly influenced by thermal effects due to the transformation heat and to a relatively large dependence of the transformation stresses on the temperature. Moreover heat production resulting from the SMA internal dissipation processes, either during martensitic transformation or reorientation has also to be taken into account.

Several theoretical and experimental studies have already been published for NiTi, CuAlNi and CuZnAl [1]. These studies concern mostly the pseudoelastic behaviour of wires as a function of the imposed strain rate and the heat transfer between the wire and its surroundings. For this one-dimensional case, assuming a homogeneous behaviour along the length of the wire and neglecting heat transfer between the wire and the grips, McCormick et al [1] have established a heat balance analysis allowing to calculate specimen temperatures which are in close agreement with experimental measurements. On the other hand Leo et al [2] assume the appearance of a single austenite-martensite interface in the wire and establish a one-dimensional model taking into account the coupling between the motion of the austenite-martensite interface and a one-dimensional heat transfer including convection, radiation and conduction along the wire and between the wire and the grips. 
The aim of this paper is to present a three-dimensional finite element simulation of thermomechanical behaviour of a polycrystalline NiTi body which is considered as a classical continuum. At each material point of the continuum one assumes at any moment a local thermodynamical constraint equilibrium state, i.e. rate dependent phenomena like viscous effects are neglected. The quasi-static mechanical behaviour of the material element is described using an elastohysteresis model depending on the current temperature of the element. Our thermomechanical model combines finally the description of the temperature dependent mechanical behaviour with a heat flow problem that includes both transformation heats and heat production resulting from internal dissipation processes. A first part of the paper deals with a brief recall of the used elastohysteresis model and with the heat production expressions deduced from this model. A second part describes the finite element model, whereas the third part is devoted to the presentation of two problems of practical importance, i.e. on the one hand the one-way shape memory effect modelling of a cantilever beam firstly deformed in martensite state and then heated, and on the other hand the influence of the deformation rate on the pseudoelastic behaviour of this beam.

\section{ELASTOHYSTERESIS MODEL AND HEAT PRODUCTION SOURCES}

The description of classical reversible thermodynamical processes representing a sequence of equilibrium state is well established since the works of Gibbs, Helmholtz and Duhem [3] and is performed by introducing thermodynamical state functions, as for example Helmholtz free energy $F$ for isothermal processes. For processes depending on one normal variable denoted by $\varepsilon$, the external action denoted $\sigma$ is calculated as the derivative of $F$ with respect to the normal variable.

Duhem considered that a study of equilibrium processes has to distinguish two other types of system, the frictional system and the system with hysteresis [3][4] for which Duhem suggested to modify the modelling of the reversible systems by incorporating another term to give:

$$
d \sigma=d\left(\frac{\partial F}{\partial \varepsilon}\right) \pm h d \varepsilon
$$

where the + is to be used when the normal variable increases and the - when it decreases. The quantity $h$ may depend upon the state of the system (through $\varepsilon$ ) and also of the external action $\sigma$. The elastohysteresis model which is used here assumes a similar decomposition of the external action with each stress contribution, denoted $\sigma_{(r)}$ and $\sigma_{(h)}$, being defined through two tensorial schemes. In order to take into account large deformation, stresses and strains are described in the body coordinate system [5][6] for which the covariant body metric tensors are $g_{i j}$ and $G_{i j}$ at initial and current time respectively. The Almansi strain tensor $\varepsilon$ and its deviatoric part $\bar{\varepsilon}$, of current covariant components $\varepsilon_{i j}=1 / 2\left(g_{i j}-G_{i j}\right)$ and $\bar{\varepsilon}_{i j}=\varepsilon_{i j}-\frac{\operatorname{trace}(\varepsilon)}{3} g_{i j}$ respectively, are then used to describe the body deformation. For initially isotropic material Favier et al [7] proposed to consider the Helmholtz free energy density as a function of the volume variation $V$ and of the second invariant $\bar{\Pi}_{\bar{\varepsilon}}$ and phase $\varphi_{\bar{\varepsilon}}$ of the deviatoric strain and to obtain the isothermal reversible stress by identifying each term of the following expression:

$$
\sigma_{(r)}^{i j} D_{i j}=\frac{\partial F}{\partial V} \dot{V}+\frac{\partial F}{\partial \bar{\Pi}_{\bar{\varepsilon}}} \dot{\bar{\Pi}}_{\bar{\varepsilon}}+\frac{\partial F}{\partial \varphi_{\bar{\varepsilon}}} \dot{\varphi}_{\bar{\varepsilon}} \quad \text { with } \dot{x}=\frac{d x}{d t} \text { and } \mathrm{i}, \mathrm{j}=1,3
$$

where $D_{i j}=I / 2 \frac{d g_{i j}}{d t}$ are the covariant components of the strain rate tensor.

The hysteresis contribution is not, as usually considered, totally dissipative. It is well known that very complex processes accompany, for example, the martensitic transformation like generation of dislocation, piling up of migrating dislocations at certain obstacles like grain boundaries, etc. This means that part of the energy due to the hysteresis stress is stored in a non-dissipative process and can interact subsequently with external energy supply depending on the stress path [8]. Based on the analysis of one-dimensional rheological 
models containing elastic and slip elements, Guélin [9] has showed that the hysteresis stress scheme has to take into account the memorization by the material of particular stress states denoted $\sigma_{(h)}^{R}$ corresponding to load reversal point. The pure hysteresis scheme is thus of discrete memory form which is written as:

$$
d \sigma_{(h)}=h\left(D, \sigma_{(h)}-\sigma_{(h)}^{R}, \omega\right)
$$

$\omega$ is the Masing parameter, with $\omega=1$ (for the first loading) or 2 afterwards.

The elastohysteresis model is built up using only external state variables, i.e. Cauchy stress, Almansi strain and temperature. This approach allows modelling of any SMA isothermal behaviour. This includes pseudoelasticity and rubber-like behaviour with only one equation whose parameters are temperature dependent. It is thus particularly interesting when a complex process like one way memory effect involving martensite reorientation and reverse transformation has to be modelled. However particular expressions for the density of Helmholtz free energy per unit of extent $F$ and $h$ have to be deduced from experimental results. Simple forms (which have to be improved taking into consideration new experimental results [10]) introducing six parameters have been proposed in the tensorial case [7]. In the isothermal one-dimensional case [10] (Fig.3), during first loading these forms lead to:

$$
\sigma_{(r)} d \varepsilon=d F=d E_{(r)}-T d S_{(r)}=\left[E_{\infty} \varepsilon+Y_{r} \text { th }\left(E_{r} \frac{\varepsilon}{Y_{r}}\right)\right] d \varepsilon \text { and } \sigma_{(h)}=Y_{0} \text { th }\left(E_{(b)} \frac{\varepsilon}{Y_{0}}\right)
$$

The hysteresis contribution is assumed temperature independent; Guélin has shown that in the case the internal energy rate $\dot{E}_{(h)}$ and the dissipated energy under heat form $\dot{Q}_{(h)}$ may be expressed as:

$$
\dot{Q}_{(h)}=\frac{1}{\omega}\left(\Delta_{R}^{t} \sigma \frac{\partial \Delta_{R}^{t} \varepsilon}{\partial t}-\Delta_{R}^{t} \varepsilon \frac{\partial \Delta_{R}^{t} \sigma}{\partial t}\right) \quad \text { with } \quad \Delta_{R}^{t} x=x(t)-x^{R}
$$

The reversible contribution is temperature dependent through $Y_{r}$ which is taken as 0 for $\mathrm{T}<\mathrm{T}_{0}$ and as a linear function of the temperature with a slope equal to $6 \mathrm{MPa} / \mathrm{K}$ for $\mathrm{T}>\mathrm{T}_{0}[1]$. The energy-entropy equilibrium can not be deduced from isothermal experiments and therefore from the determination of the Helmholtz energy alone. It would necessary to perform adiabatic tests for which $\mathbf{o}_{(\eta)}^{i j} D_{i j}=\dot{F}+T \dot{S}_{(r)}$ allowing thus the determination of $\dot{Q}_{(r)}=T \dot{S}_{(r)}$

\section{FINITE ELEMENT MODEL}

We take into account at the same time mechanical and thermal problems. Assumptions and numerical model for the mechanical part have already been presented in [12][13]. We are only going to recall the main ideas. For the presented test, we use 3-dimensional elements with $\mathrm{C}^{0}$ isoparametric interpolation, quadratic hexahedrons, with a Gauss numerical integration which uses 8 integration points. Concerning kinematics and behaviour law, the study is non-linear. Equilibrium equations have been approached using virtual power principle. Non-linearity of the behaviour law has been approached by the Newton method. We consider an implicit time formulation. For time increment, degrees of freedom rate is constant. Using implicit formulation for the time discretization, non-linearity of global equilibrium has been solved by the Newton-Raphson incremental method. So we have to calculate all the derivatives and more particularly for the both parts of the behaviour law, i.e. hyperelastic and hysteresis. The hyperelastic stress contribution is defined by [12]:

$$
F=\frac{K_{r} \ln ^{2} V}{6}+\frac{Q_{a r}^{2}}{2 \mu_{r}} \ln \left[\cosh \left(\frac{2 \mu_{r}}{Q_{a r}(T)}\right) \sqrt{2 \bar{\Pi}_{\bar{\varepsilon}}^{-}}\right]+2 \mu_{\infty} \bar{\Pi}_{\bar{\varepsilon}}
$$

$\mathrm{K}_{\mathrm{r}}, \mu_{\mathrm{r}}$ and $\mu_{\infty}$ being constant parameters and $\mathrm{Q}_{\mathrm{or}}$ being temperature dependent. The above hysteresis behaviour constitutive equation is defined by a rate form [12]:

$$
\begin{gathered}
\frac{\partial}{\partial t}\left(\Delta_{R}^{t} S_{j}^{\prime i}\right)=2 \mu_{h} \bar{D}_{j}^{i}+\beta_{4} \bar{\phi} \Delta_{R}^{t} S_{j}^{\prime i} \text { with } \Delta_{R}^{t} S_{j}^{i j}=S_{j}^{\prime i}(t)-S_{j}^{\prime i}\left(t_{R}\right) \text { and } S_{(h)}^{i j}=S_{k}^{{ }^{i} i} g^{k j}+S_{k}^{j j} g^{k i} \\
\bar{\phi}=\Delta_{R}^{t} S_{j}^{\prime i} \bar{D}_{i}^{j} \text { and } \beta_{4}=\frac{-2 \mu_{h}}{\omega^{2} Q_{h}^{2}}
\end{gathered}
$$


$S_{(j)}$ represents the Cauchy stress tensor deviatoric part. This leads to the global behaviour: $\sigma=\sigma_{r}+S_{h}$. Thermal approach is carried out by the Galerkine method, likewise for mechanics, with temperature as nodal unknown and a temperature virtual rate equilibrium. So we obtain the following weak formulation:

$$
\int_{D}\left[\left(\boldsymbol{K}_{c} \cdot \vec{\nabla} T\right) \cdot \vec{\nabla} \stackrel{*}{T}+\left(\boldsymbol{r}-\rho C_{p} \dot{T}\right) \stackrel{*}{T}\right] d V-\int_{\partial D}\left(\boldsymbol{K}_{c} \cdot \vec{\nabla} T\right) \vec{n} \stackrel{*}{T} d S=0
$$

with T: temperature, $\stackrel{*}{T}$ : virtual temperature rate and $\mathrm{r}=\dot{Q}_{(h)}+\dot{Q}_{(r)}$

Calculations are correct with a symmetrical stiffness matrix, in order to limit the stocking zone.

Boundary conditions, implanted to date, are of temperature or imposed flux type. Temperature discretization and quadrature are identical to those used in mechanics. Transitional phenomena have been approached by the finite differences method with the implicit scheme. Thermal and mechanical coupling is taken into account in two points. On the one hand, behaviour law depends on temperature, through the hyperelastic potential by the $Q_{o r}$ coefficient; on the other hand, mechanical deformation induces a thermal source through $r$ which represents the 3-dimensional power produced by mechanical deformations.

\section{EXPERIMENT}

a) Firstly, the one-way memory effect of a cantilever beam has been investigated. The mesh consists of an optimum numbers of elements adjusted during classical bending test: ten length elements, two quadratic thickness elements and two width elements. Dimensions of the beam: $1 \times \mathrm{w} \times \mathrm{h}=200 \times 40 \times 10 \mathrm{~mm}$.

Values relating to the mechanical behaviour are :

$$
\mu_{\infty}=1000 \mathrm{MPa} \quad \mu_{0}=15000 \mathrm{MPa} \quad K_{r}=95000 \mathrm{MPa} \quad Q_{h}=100 \mathrm{MPa}
$$

A bending test with load and unload in martensitic phase $(\Delta \mathrm{T}=0)$ has been carried out. Residual displacements are then maintained at the end of the beam, and the temperature of the beam has been risen (Fig. 1) slowly such as at each time temperature is uniform and equal to the surrounding one.
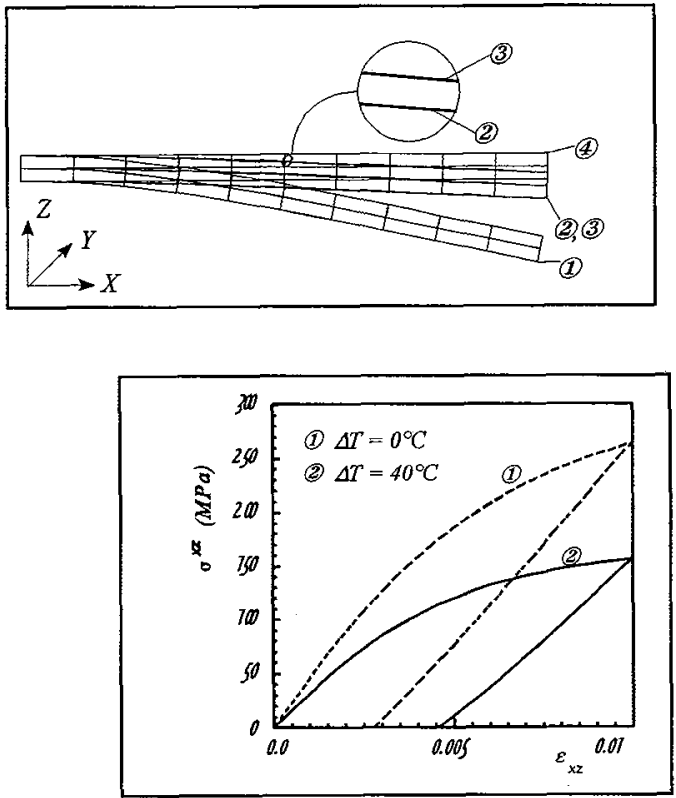

Figure 2: Variation of the behaviour law with temperature
Figure 1: Beam shapes during one-way memory effect simulation

(1) Maximum deformed shape, $\Delta \mathrm{T}=0^{\circ} \mathrm{C}, \Delta \mathrm{z}_{\mathrm{B}}=32 \mathrm{~mm}$

(2) Residual deformed shape, $\Delta \mathrm{T}=0^{\circ} \mathrm{C}, \Delta \mathrm{z}_{\mathrm{B}}=7.5 \mathrm{~mm}$

(3) Deformed shape after heating, $\Delta \mathrm{T}=40^{\circ} \mathrm{C}$, maximum difference between (2) and (3) for $\mathrm{z}$ deflection $\approx 610^{-3} \mathrm{~mm}$ at $\mathrm{x}=100 \mathrm{~mm}$ (4) Initial beam shape

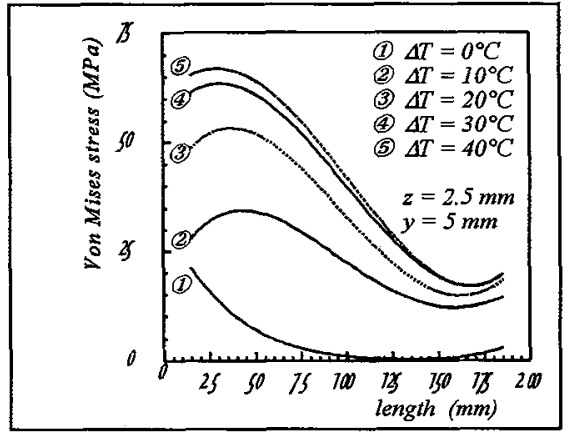

Figure 3: Von Mises stress repartition during the change from martensite to austenite 
Here $Q_{o r}$ allows to model the change from martensite to austenite such as: $Q_{o r}=0.1+200 \tanh \left(\frac{\Delta T}{40}\right)$ (Fig. 2). During the temperature rise, stress repartition changes (Fig. 3) and leads to beam reaction (Fig. 4).

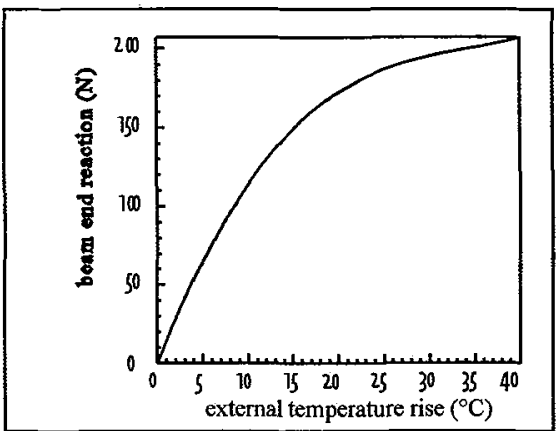

Figure 4: Beam end reaction during the change from martensite to austenite

b) Secondly the two following tests concern the pseudoelastic behaviour. At first we identified the thermal power due to the mechanical deformation during a simple near-adiabatic tensile test. $\dot{Q}_{(\lambda)}$ has been taken as proportional to $\frac{\partial}{\partial \gamma} \sqrt{2 \pi_{\varepsilon}^{-}}$so as to obtain a rise of temperature of about $10^{\circ} \mathrm{C}$ for $6 \%$ elongation [1]; in this simulation $\dot{Q}_{(2)}$ is neglected. From a numerical aspect, this simple test allows to bring to the fore several calculation particularities. In order to avoid an important external thermal loss, a very short time has to be used. However if this time is too short, the thermal stiffness is entirely dominated by the specific heat and in this case using 4 integration points, the thermal stiffness becomes singular. Due to the approximation errors, some acceptable temperatures have been obtained at the integration points. This is not the case at the nodes.

Test conditions: material characteristics are the same as for the first test and the initial temperature is $\Delta \mathrm{T}=$ $40^{\circ} \mathrm{C}$; dimensions of the parallelepipedic tensile sample $(100 \times 40 \times 20 \mathrm{~mm}), 6 \mathrm{~mm}$ elongation with an imposed displacement for one of the two $40 \times 20$ faces. Total time for the test: $6,910^{-5} \mathrm{~s}$. Thermal parameters: $\rho=6.510^{-3} \mathrm{~g} / \mathrm{mm}^{3} \quad K_{c}=210^{-2} \mathrm{~W} / \mathrm{mm} / K \quad C_{p}=0.53 \mathrm{~J} / \mathrm{g} / \mathrm{K}$. For both of the $40 \times 20$ faces, ambient temperature has been maintained so as to impose temperature boundary conditions. However test time is sufficiently short to avoid an important out of beam energy. Concerning the rest of the boundary a null flux has been imposed (adiabatic conditions).

We were interested, secondly, in the pseudoelastic behaviour of a cantilever beam (the same as for the first test in a)). This part is aimed at showing the temperature rise in the case of a rapid loading test (adiabatic conditions). Two thickness quadratic elements are sufficient, but we used three to improve visualisation of the temperature evolution calculated at integration points.

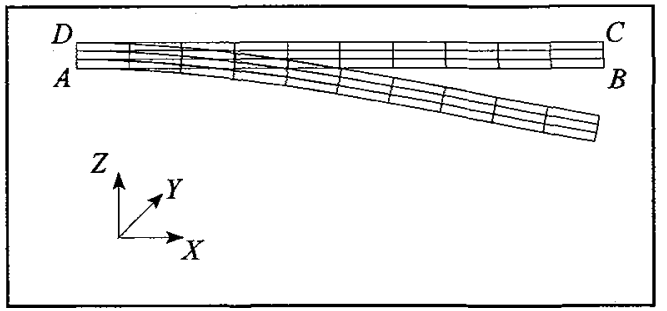

Boundary conditions:

$C B \quad: 32 \mathrm{~mm}$ imposed displacement according to $\mathrm{z}$
$\mathrm{AD}, \mathrm{BC}:$ : ambient temperature
$\mathrm{AD}:$ clamped $\mathrm{U}_{1}=\mathrm{U}_{2}=\mathrm{U}_{3}=0$
$\mathrm{AB}, \mathrm{DC}:$ null flux

Figure 5: Initial and deformed shape for an adiabatic bending

Calculation is carried out in large transformations (large displacements, large deformations). Material is identical to the one used during the tensile test. 


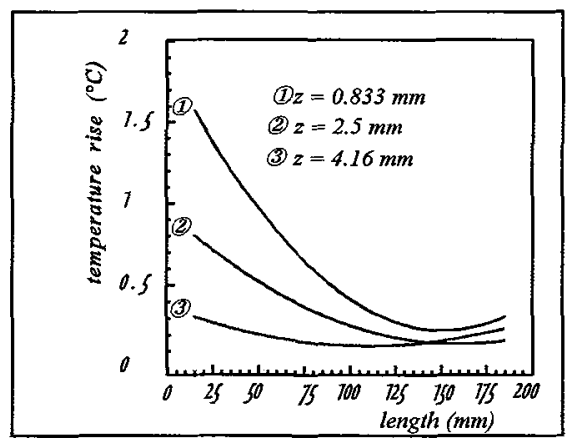

Figure 6: Temperature evolution along beam length

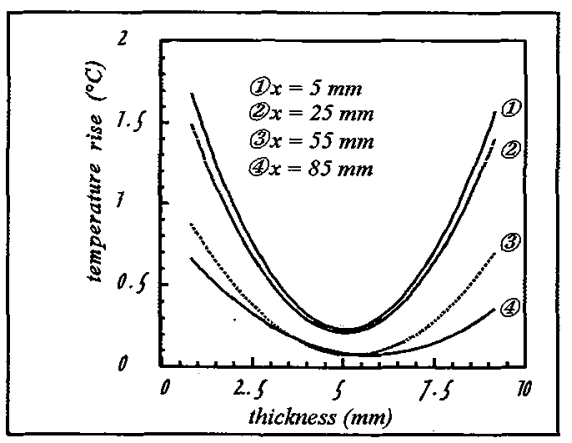

Figure 7: Temperature evolution along cross section

Figure 6 shows the temperature evolution along the beam length in relation to the thickness position. Figure 7 shows the temperature evolution of the cross section. We note a symmetrical behaviour near the clamp with a maximum temperature rise about $1.5^{\circ} \mathrm{C}$. At the middle of the beam $(x=85 \mathrm{~mm})$, this symmetry is not conserved. We observe the same dissymmetry concerning the Von Mises stress, which explains the previous result.

\section{CONCLUSION}

The aim of this work is to present a modelling of the thermomechanical behaviour of NiTi shape memory alloys. The proposed model is a 3-dimensional elastohysteresis type. Calculations are carried out by 3dimensional finite element, taking into account large deformations and displacements, thermal transitional equilibrium and thermomechanical couplings. Two non-homogeneous tests have been presented. The first one concerns one way shape memory effect of a cantilever beam. The second one shows temperature repartition during pseudoelastic bending of this beam. In both cases obtained results are coherent with experimental observation.

\section{REFERENCES}

[1] McCormick P.C., Liu Y. and Miyazaki S., Mat. Sci. and Eng., A167, 51-56, 1993

[2] Leo P.H., Shield T.W. and Bruno O.P., Acta Metall. Mater., Vol.41, N8, 2477-2485, 1993

[3] Duhem P.M., "L'évolution de la mécanique", 1903, or "The evolution of mechanics", Sisjhoff \& Noordhoff, Maryland, 1980

[4] Favier D., Guélin P., Cammarano R., in proc."7th Int. Symposium on Magnetic Anisotropy \& Coercivity in RE-TM Alloys", Canberra, Australia, 137-150, 1992

[5] Lodge A.S., "Body tensor fields in Continuum Mechanics", Academic Press, New-York, 1974

[6] Pégon P., Guélin P., Int. J. for Num. Meth. in Eng., Vol. 22, 521-545, 1986

[7] Favier D., Guélin P. and Pégon P., Mat. Sci. Forum, Vol. 56-58, 569-564, 1990

[8] Liu Y., Favier D., Orgeas L., Icomat'95

[9] Guélin P., Journal de Mécanique, Vol.19, N2, 217-247, 1980

[10] Orgeas L., Favier D., Icomat'95

[11] Manach P.Y., Favier D., Icomat'92, Monterey, California, 941-945, 1993

[12] Rio G., Manach P.Y., Favier D., Archives of Mechanics, Vol.3, 1995

[13] Manach P.Y., Favier D., Rio G., International Seminar Mecamat'95 "Mechanisms and Mechanics of Solid-Solid Phase Transformation", Journal de Physique (in press) 